\title{
Two types of human Th17 cells with pro- and anti-inflammatory properties and distinct roles in autoinflammation
}

\author{
R Noster ${ }^{1,2}$, H de Koning ${ }^{3}$, F Sallusto $^{4}$, C Zielinski $^{1 *}$ \\ From 8th International Congress of Familial Mediterranean Fever and Systemic Autoinflammatory Diseases \\ Dresden, Germany. 30 September - 3 October 2015
}

\section{Introduction}

Th17 cells are known to be crucial mediators of autoimmune inflammation. However, two distinct types of Th17 cells have recently been described, which differed in their ability to coproduce IL-10 or IFN-g due to differential polarizations requirements for IL-1b. Whether these distinct Th17 phenotypes translate into distinct Th17 cell functions and whether this has implications for human health or disease has not been addressed yet.

\section{Objectives}

We hypothesized that IL-1b independent IL- $10^{+}$Th17 cells have anti-inflammatory functions whereas IL-1b dependent IL-10- Th17 cells are pro-inflammatory. Considering the crucial role of IL-1b in the pathogenesis of autoinflammatory syndromes, we hypothesized an IL-1b mediated loss of anti-inflammatory Th17 cell functions in Schnitzler Syndrome, an autoinflammatory disease.

\section{Methods}

To assess pro- versus anti-inflammatory Th17 cell functions we performed suppression assays and tested the effects of IL-1b dependent and independent Th17 subsets on modulating pro-inflammatory cytokine secretion by monocytes. Schnitzler Syndrome patients were analyzed for changes in Th17 cell functions before and after therapy with IL-1b depleting drugs.

\section{Results}

IL- $10^{+}$Th17 cells, which differentiated independently of IL-1b, have regulatory functions similar to Treg cells while IL-1b dependent IL-10- Th17 cells have not. Both Th17 cell subsets differ in their ability to suppress $\mathrm{T}$ cell proliferation as well as in their ability modulate pro-inflammatory cytokine production by antigen presenting cells. In Schnitzler Syndrome, an autoinflammatory syndrome, overproduction of IL-1b translates into pro-inflammatory Th17 cell functions, which can be reversed by anti-IL-1b treatment.

\section{Conclusion}

Th17 cells are not per se pro-inflammatory but can also have anti-inflammatory IL-10 mediated functions if generated independently of IL-1b. Our data introduce Th17 cell subsets as novel players in autoinflammation and thus novel therapeutic targets in autoinflammatory syndromes including other IL-1b mediated diseases. This demonstrates for the first time alterations in the adaptive immune system in autoinflammatory syndromes.

\section{Authors' details \\ ${ }^{1}$ Institute for Medical Microbiology, Immunology and Hygiene, München, Germany. ${ }^{2}$ Charité-Universitätsmedizin Berlin, Berlin, Germany. ${ }^{3}$ Radboud University Nijmegen Medical Centre, Nijmegen, Netherlands. ${ }^{4}$ nstitute for Research in Biomedicine, Bellinzona, Switzerland.}

Published: 28 September 2015

doi:10.1186/1546-0096-13-S1-049

Cite this article as: Noster et al:: Two types of human Th17 cells with pro- and anti-inflammatory properties and distinct roles in autoinflammation. Pediatric Rheumatology 2015 13(Suppl 1):049.

${ }^{1}$ Institute for Medical Microbiology, Immunology and Hygiene, München,

Germany

Full list of author information is available at the end of the article 